\title{
Intermolecular dynamics of water: Suitability of reactive interatomic potential
}

\author{
Dipak Prasad, ${ }^{\dagger}$ Nilanjan Mitra, ${ }^{*, \ddagger}$ and Sanjoy Bandyopadhyay, ${ }^{*}$ \\ $\dagger$ Department of Civil Engineering, Indian Institute of Technology Kharagpur, India \\ $\ddagger$ Department of Civil Engineering $\&$ Centre for Theoretical Studies, Indian Institute of \\ Technology Kharagpur, India \\ \Department of Chemistry $\&$ Centre for Theoretical Studies, Indian Institute of Technology \\ Kharagpur, India \\ E-mail: Nilanjan@civil.iitkgp.ac.in; sanjoy@chem.iitkgp.ac.in
}

\section{Review of interatomic potentials}

\section{TIP4P $/ 2005 f$}

TIP4P flexible model ${ }^{1}(\mathrm{TIP} 4 \mathrm{P} / 2005 \mathrm{f})$ is extension of TIP4P model $^{2}(\mathrm{TIP} 4 \mathrm{P} / 2005)$ with relaxation incorporated for intramolecular degree of freedom. TIP4P/2005f model consists of 4-point interaction site, $\mathrm{H}-\mathrm{O}-\mathrm{H}$ along with $\mathrm{M}$ site lying on bisector of $\mathrm{H}-\mathrm{O}-\mathrm{H}$ angle. Here two positive and one negative charges are placed at two hydrogens and at M-site respectively. Only oxygen is considered as Lennard-Jones interaction site. Hence LJ interaction between two water molecule is defined as

$$
U=4 \epsilon\left[\left(\frac{\sigma}{r_{O O}}\right)^{12}-\left(\frac{\sigma}{r_{O O}}\right)^{6}\right]
$$


Whereas electrostatic interaction between charge sites are given by coulomb's inverse square law

$$
U=\frac{1}{4 \pi \epsilon_{0}} \sum_{i=1}^{n-1} \sum_{j>i}^{n} \frac{q_{i} q_{j}}{r_{i j}}
$$

The cutoff for long range LJ interaction was $8.5 \AA$. Similarly electrostatic interaction is computed with Ewald summation technique using real space cutoff of $8.5 \AA$. Potential energy due to intramolecular degrees of freedom consists of two $\mathrm{O}-\mathrm{H}$ bonds and one $\mathrm{H}-\mathrm{O}-\mathrm{H}$ bond angle. Interactions for $\mathrm{O}-\mathrm{H}$ bond and $\mathrm{H}-\mathrm{O}-\mathrm{H}$ bond angle are expressed as Morse potential and harmonic function respectively.

$$
\begin{gathered}
U\left(r_{O H}\right)=D_{r}\left[1-\exp \left(-\beta\left[r_{O H}-r_{e q}\right]\right)\right]^{2} \\
U(\theta)=\frac{1}{2} K_{\theta}\left(\theta-\theta_{e q}\right)^{2}
\end{gathered}
$$

where $\mathrm{r}_{e q}$ and $\theta_{e q}$ are equilibrium bond distance and bond angle respectively. Distance of negative charge site $\mathrm{M}$ from Oxygen is defined in terms of two O-H bond length and $\mathrm{H}-\mathrm{O}-\mathrm{H}$ bond angle $\theta$ as follows

$$
d_{O M}=d_{O M}^{r e l}\left(r_{O H_{1}}+r_{O H_{2}}\right) \cos (\theta / 2)
$$

The values of different governing parameters are as follows: $\epsilon=0.1852 \mathrm{Kcal} / \mathrm{mol}, \quad \sigma=3.1644 \AA$, $q_{H}=0.5564 e$, $d_{O M}^{r e l}=0.13194, \quad D_{r}=432.581 \mathrm{~kJ} / \mathrm{mol}, \quad r_{e q}=0.9419 \AA$, $\theta_{e q}=107.4^{\circ}, \quad \beta=2.287 \AA^{-1}, \quad \mathrm{~K}_{\theta}=367.810 \mathrm{~kJ} /\left(\mathrm{mol} \mathrm{rad}^{2}\right)$.

For a model like TIP4P/2005f there exist conflict between prediction of melting temperature and enthalpy of vaporization. However one of the key parameter in prediction of different properties of water is temperature of maximum density $\left(\mathrm{T}_{m d}\right)$. This $\mathrm{T}_{m d}$ along with different critical points in phase diagram are taken as governing property for parametrization of TIP4P/2005f which also keeps balance between melting point and enthalpy of vaporiza- 
tion.

\section{REAX/2017}

Reax/2017 water model $^{3}$ is result of subsequent development of Reax/2010 water model ${ }^{4-6}$ in order to predict the correct density of bulk water as well as order of diffusion constant of water, hydronium and hydroxide ion $\left(\mathrm{H}_{2} \mathrm{O}<\mathrm{OH}^{-}<\mathrm{H}_{3} \mathrm{O}^{+}\right)$. Here energy and structure of different clusters were parametrized against data from QM simulations while density and diffusion coefficients were fitted against experimental values. The whole paramatrization lies around least square method.

$$
\text { error }=\sum_{i}^{n}\left(\frac{x_{i, r e f}-x_{i, r e a x}}{\sigma_{i}}\right)^{2}
$$

where $\sigma_{i}$ is weightage given to ith data, $x_{i, r e f}$ and $x_{i \text {,reax }}$ are data from QM or experimental investigation and Reax/2017 forcefield respectively. Different properties accounted for fitting are variation in energy for $\mathrm{O}-\mathrm{H}$ bond length, $\mathrm{H}-\mathrm{O}-\mathrm{H}$ bond angle, binding energy of water dimer with different symmetries etc.

The forcefield parameters are related with various properties according to dependence of energy on different interactions. For a standard Reax model energy of system can be expressed as

$$
\begin{aligned}
E & =E_{\text {bond }}+E_{\text {angle }}+E_{\text {dihedral }}+E_{\text {over }}+E_{\text {under }} \\
& +E_{\text {lonepair }}+E_{\text {vanderwaal }}+E_{\text {electrostatic }}+E_{H B}
\end{aligned}
$$

where energy includes contribution from bond length, bond angle, torsional angle, overcoordination or undercoordination of atoms, lonepair energy, Van der Waals, electrostatic and intermolecular hydrogen bonds. Some of the energy forms had been defined as follows

$$
E_{b o n d}=-D_{e} B O_{i j} \exp \left[p_{b e, 1}\left(1-B O_{i j}^{p_{b e, 1}}\right)\right]
$$




$$
\begin{gathered}
E_{\text {angle }}=f\left(B O_{i j}\right) f\left(B O_{j k}\right) g\left(\Delta_{j}\right) \\
\times\left\{k_{a}-k_{b} \exp \left[-k_{b}\left(\Theta_{o}-\Theta_{i j k}\right)^{2}\right]\right\} \\
E_{\text {overcoordination }}=p_{\text {over }} \Delta_{i}\left(\frac{1}{1+\exp \left(\lambda_{6} \Delta_{i}\right)}\right) \\
E_{\text {undercoordination }}=-p_{\text {under }} \frac{1-\exp \left(\lambda_{7} \Delta_{i}\right)}{1+\exp \left(-\lambda_{8} \Delta_{i}\right)} \\
E_{v d w}=D_{i j}\left[\exp \left\{\alpha_{i j}\left(1-\frac{h\left(r_{i j}\right)}{r_{v d w}}\right)\right\}\right] \\
-2 D_{i j}\left[\exp \left\{\frac{1}{2} \alpha_{i j}\left(1-\frac{h\left(r_{i j}\right)}{r_{v d w}}\right)\right\}\right] \\
\left.E_{\text {electrostatic }}=C \frac{q_{i} q_{j}}{\left[r_{i j}^{3}+\left(1 / \gamma_{i j}\right)^{3}\right]}\right]^{1 / 3}
\end{gathered}
$$

Here bond order is calculated as

$$
B O_{i j}=B O_{i j}^{\prime} \Psi\left(\Delta_{i}^{\prime}, \Delta_{j}^{\prime}, B O_{i j}^{\prime}\right)
$$

where

$$
\begin{array}{r}
B O_{i j}^{\prime}=\exp \left[p_{b o, 1}\left(\frac{r_{i j}}{r_{o}}\right)^{p_{b o, 2}}\right]+ \\
\exp \left[p_{b o, 3}\left(\frac{r_{i j}^{\pi}}{r_{o}}\right)^{p_{b o, 4}}\right]+\exp \left[p_{b o, 5}\left(\frac{r_{i j}^{\pi \pi}}{r_{o}}\right)^{p_{b o, 6}}\right]
\end{array}
$$

The three terms in above expression responds to contribution from $\sigma$, first $\pi$ and second $\pi$ bond respectively. $B O_{i j}^{\prime}$ and $B O_{i j}$ are uncorrected and corrected bond order respectively. 
Similarly deviation from actual valency can be defined as

$$
\begin{aligned}
\Delta_{i}^{\prime} & =\sum_{j=1}^{n b o n d} B O_{i j}^{\prime}-V a l_{i} \\
\Delta_{i} & =\sum_{j=1}^{n b o n d} B O_{i j}-V a l_{i}
\end{aligned}
$$

Full detail of governing parameters can be found in following literatures ${ }^{7}$. Reax $/ 2017 \mathrm{had}$ been proved to be excellent in prediction of binding energy as well as proton transfer mechamism in $\mathrm{H}_{3} \mathrm{O}^{+}\left(\mathrm{H}_{2} \mathrm{O}\right)$ n or $\mathrm{OH}-\left(\mathrm{H}_{2} \mathrm{O}\right)$ n cluster.

\section{References}

(1) González, M. A.; Abascal, J. L. A flexible model for water based on TIP4P/2005. The Journal of Chemical Physics 2011, 135, 224516.

(2) Abascal, J. L.; Vega, C. A general purpose model for the condensed phases of water: TIP4P/2005. The Journal of Chemical Physics 2005, 123, 234505.

(3) Zhang, W.; Van Duin, A. C. Second-Generation ReaxFF Water Force Field: Improvements in the Description of Water Density and OH-Anion Diffusion. The Journal of Physical Chemistry B 2017, 121, 6021-6032.

(4) Raymand, D.; van Duin, A. C.; Spångberg, D.; Goddard III, W. A.; Hermansson, K. Water adsorption on stepped $\mathrm{ZnO}$ surfaces from MD simulation. Surface Science 2010, $604,741-752$.

(5) Fogarty, J. C.; Aktulga, H. M.; Grama, A. Y.; Van Duin, A. C.; Pandit, S. A. A reactive molecular dynamics simulation of the silica-water interface. The Journal of Chemical Physics 2010, 132, 174704.

(6) van Duin, A. C.; Zou, C.; Joshi, K.; Bryantsev, V.; Goddard, W. A. A Reaxff reactive 
force-field for proton transfer reactions in bulk water and its applications to heterogeneous catalysis. Computational Catalysis 2013, 14, 223.

(7) Van Duin, A. C.; Dasgupta, S.; Lorant, F.; Goddard, W. A. ReaxFF: a reactive force field for hydrocarbons. The Journal of Physical Chemistry A 2001, 105, 9396-9409. 